\title{
CONJUGATE LOCALLY CONVEX SPACES. II
}

\author{
BY \\ V. KRISHNAMURTHY
}

1. Introduction. In this paper we continue the project started in [4]-we shall refer to this paper as (I) in the sequel-of generalizing the results of Dixmier [1] from the setting of Banach spaces to that of locally convex linear Hausdorff spaces (hereafter to be abbreviated as 1.c. spaces). One of his main results, namely, the characterization of conjugate Banach spaces [1, p. 1069, Theorem 17], was generalized by the author to 1.c. spaces in (I). But in Dixmier's characterization he used the concept of the characteristic of a subspace in the dual of a Banach space. This concept was not generalized to 1.c. spaces in (I). We propose to do this in this paper.

We use the terminology of (I) which is mainly that of Köthe [3]. Recall in particular that if $\langle F, G\rangle$ is a dual system, $\tau_{s}(G, F)$ or $\tau_{s}(G)$ (when $F$ is clear from the context) denotes the weak topology of $F$ defined by the elements of $G$. Let $E$ be a Banach space and $E^{\prime}$ its dual. Let $S_{\lambda}$ be the closed ball of radius $\lambda$ in $E^{\prime}$ and $\Sigma$ the unit ball in $E$. The characteristic of a subspace $V$ of $E^{\prime}$ is then given, according to Dixmier [1, Definition 2 and Theorems 7, 8 and 9, pp. 1062-1064], by any of the following four equivalent expressions:

(1.1) $\max \left\{\lambda \geqq 0: V \cap S_{1}\right.$ is $\tau_{s}(E)$-dense in $\left.S_{\lambda}\right\}$,

(1.2) $\inf \left\{\sup _{f \in V \cap S_{1}}|f(x)| /\|x\|: x \neq 0, x \in E\right\}$,

(1.3) $1 / \sup \left\{\|x\|: x \in \tau_{s}(V, E)\right.$-closure of $\left.\Sigma\right\}$,

(1.4) $\inf \left\{\|x+z\| /\|x\|: z \in V^{\perp}, x \in E, x \neq 0\right\}$,

$V^{\perp}$ being the orthogonal complement of $V$ in $E^{\prime \prime}$.

We shall refer to the real number defined above as the Dixmier characteristic $D(V)$ of $V$.

In the rest of the paper let $E[\tau]$ and $E^{\prime}$ stand for an 1.c. space and its dual. In Dixmier's characterization of conjugate Banach spaces, an essential role was played by subspaces of characteristic one in the dual. In the generalization to 1.c. spaces mentioned above, the analogous role was taken by what we called duxial subspaces, defined as follows. A linear subspace $V$ of $E^{\prime}$ is said to be duxial if every convex $\tau_{s}(E)$-compact subset of $E^{\prime}$ is contained in the $\tau_{s}(E)$-closure of a bounded subset of $V\left[\tau_{b i}\left(E, E^{\prime}\right)\right]$, that is, $V$ with the topology induced by $E^{\prime}\left[\tau_{b}(E)\right]$. It is easy to see that a duxial subspace in the dual of a Banach space is nothing but a subspace of characteristic greater than zero.

We define in this paper two different characteristics $\alpha(V)$ and $\beta(V)$ for a subspace $V$ in the dual $E^{\prime}$ of any l.c. space $E$ using (1.1) as analogy. Both the expressions

Received by the editors December 1, 1966. 
reduce to the Dixmier characteristic $D(V)$ when $E$ is a normed linear space. In generalizing the different expressions for $D(V)$ we find that (1.2) generalizes to both $\alpha(V)$ and $\beta(V)$ (cf. Theorem 6) but we have no generalization of (1.4) for $\alpha(V)$ (cf. Theorem 7). The proofs of these are patterned after those of Dixmier. But it is in the generalization of (1.3) that we really find the niceties of the investigation. The analogues of (1.3) and of its consequences, presented in Theorems $1,2,4$ and 5, come off with such an elegance as would allow us to claim that a proper setting for the discussion of the characteristic is that of a 1.c. space and not that of a normed linear space. Our Theorem 4 examines the relationship between the concepts of duxiality and that of characteristic one. As an application we prove that $E$ is always of characteristic one in $E^{\prime \prime}$ (Dixmier had this result for Banach spaces) whereas $E$ is duxial in $E^{\prime \prime}$ if and only if (cf. Theorem 5) $E^{\prime}\left[\tau_{b}(E)\right]$ is a Mackey space.

2. Some remarks on notation. If $A \subset E^{\prime}, A^{0}$ will denote the polar of $A$ in $E^{\prime \prime}$, the bidual of $E$, and $A_{0}$ will denote the polar of $A$ in $E$. If $A$ is convex, $\left(A_{0}\right)^{0}$, which is the $\tau_{s}\left(E, E^{\prime}\right)$-closure of $A$ in $E^{\prime}$, will be denoted simply by cl $A$. For $B \subset E, B^{0}$ will denote the polar of $B$ in $E^{\prime} . \mathscr{U}$ will stand for a fundamental system of closed absolutely convex neighbourhoods of zero in $E[\tau]$. If $U \in \mathscr{U}, p(U, x)$ will be the seminorm of $x \in E$ defined by $U . \mathscr{M}$ and $\mathscr{B}$ will stand for the class of all absolutely convex bounded closed subsets of $E[\tau]$ and $E^{\prime}\left[\tau_{b}(E)\right]$ respectively. For any $M \subset E$ and $U \in \mathscr{U}, p(U, M)$ will stand for $\sup \{p(U, x): x \in M\}$ which is the same as $\inf \{\rho \geqq 0: M \subset \rho U\}$. Clearly $p(U, M)<\infty$ for all $U$ if and only if $M$ is bounded. The same interpretation is to be given for $p\left(B_{0}, M\right)$, where $B \in \mathscr{B}$ and $M \subset E$ and for $p\left(M^{0}, B\right)$ where $M \in \mathscr{M}$ and $B \in \mathscr{B}$.

3. Definition of characteristic. Given a subspace $V$ of $E^{\prime}$, define the following numbers:

(3.1) $\alpha(V, U, M)=\max \left\{\rho \geqq 0: \operatorname{cl}\left(V \cap M^{0}\right) \supset \rho U^{0}\right\}$ for every $U \in \mathscr{U}$ and $M \in \mathscr{M}$ such that $p(U, M) \neq 0$.

(3.2) $\alpha(V, U)=\inf \{\alpha(V, U, M) p(U, M): M \in \mathscr{M}, p(U, M) \neq 0\}$ for every $U \in \mathscr{U}$.

(3.3) $\alpha(V)=\inf \{\alpha(V, U): U \in \mathscr{U}\}$.

(3.4) $\beta(V, U, B)=\max \left\{\rho \geqq 0: \operatorname{cl}\left(V \cap U^{0}\right) \supset \rho B\right\}$ for every $U \in \mathscr{U}$ and $B \in \mathscr{B}$.

(3.5) $\beta(V, U)=\beta\left(V, U, U^{0}\right)$.

(3.6) $\beta(V)=\inf \{\beta(V, U): U \in \mathscr{U}\}$.

$\alpha(V)$ and $\beta(V)$ are called the $\alpha$-characteristic and the $\beta$-characteristic of $V$.

REMARK 1. Whenever $p(U, M)=\lambda>0$, we have $M \subset \lambda U$, so that $(1 / \lambda) U^{0} \subset M^{0}$. This shows, in virtue of the definition of $\lambda$, that $1 / \lambda U^{0}$ is the largest multiple of $U^{0}$ that is contained in $M^{0}$. So, whenever $\operatorname{cl}\left(V \cap M^{0}\right) \supset \rho U^{0}$, it follows that $\rho \leqq 1 / \lambda$. In other words, $0 \leqq \alpha(V, U, M) p(U, M) \leqq 1$. This leads in turn to $0 \leqq \alpha(V, U) \leqq 1$ and to $0 \leqq \alpha(V) \leqq 1$.

REMARK 2 . It is easily seen that $0 \leqq \beta(V, U) \leqq 1$ and $0 \leqq \beta(V) \leqq 1$. 
REMARK 3. Suppose $E$ is a normed linear space, $\Sigma$ its unit ball and $S_{1}$ that of $E^{\prime}$. Then $\alpha(V, \Sigma, \Sigma)=D(V)$. Take $U=r \Sigma$ and $M=s \Sigma$. Then $\alpha(V, U, M)=(r / s) \alpha(V, \Sigma, \Sigma)$. But $p(U, M)$ would now be $s / r$. So $\alpha(V, U, M) p(U, M)$ would again be $\alpha(V, \Sigma, \Sigma)$. Thus in the case of a normed linear space $\alpha(V, U, M) p(U, M)$ is constant for all $U$ and $M$ and is equal to the Dixmier characteristic of $V$. So $\alpha(V, U)$ and also $\alpha(V)$ are equal to the Dixmier characteristic of $V$.

REMARK 4. In the case of a normed linear space $\beta(V, U)$ is constant for all $U$ and is equal to the Dixmier characteristic of $V$. Hence $\beta(V)$ is also the Dixmier characteristic. In view of Remark 3 this means that $\alpha(V)$ and $\beta(V)$ both coincide for a normed linear space with $D(V)$.

4. Main Theorems. In all that follows, $V$ denotes a $\tau_{s}(E)$-dense subspace of $E^{\prime}$ and $U$ (respectively, $M$ and $B$ ) denotes a member of $\mathscr{U}$ (respectively, $\mathscr{M}$ and $\mathscr{B}$ ). For $M \in \mathscr{M}$, unless otherwise mentioned, $\bar{M}$ denotes the $\tau_{s}(V, E)$-closure of $M$ in $E$.

TheOREM 1. Let $p(U, M) \neq 0$. Consider the following statements:

(a) $p(U, \bar{M})<\infty$;

(b) $\alpha(V, U, M)>0$;

(c) $\alpha(V, U, M)=1 / p(U, \bar{M})$.

Then $(\mathrm{a}) \Leftrightarrow(\mathrm{b})$ and each implies (c).

Proof. The theorem is an immediate consequence of the following two lemmas.

Lemma 1. If $p(U, M) \neq 0$ and $p(U, \bar{M})<\infty$ then $\alpha(V, U, M) \geqq 1 / p(U, \bar{M})$.

LEMMA 2. If $p(U, M) \neq 0$ and $\alpha(V, U, M)>0$ then $\alpha(V, U, M) \leqq 1 / p(U, \bar{M})$.

Proof of Lemmas 1 and 2. $\bar{M}$, being the bipolar of $M$ for the dual system $\langle E, V\rangle$, is equal to $\left(V \cap M^{0}\right)_{0}$. Hence $(\bar{M})^{0}=\operatorname{cl}\left(V \cap M^{0}\right)$. Let us write $\alpha=\alpha(V, U, M)$.

Now if $\bar{M} \subset \rho U$ for some $\rho>0$, then $\operatorname{cl}\left(V \cap M^{0}\right) \supset(1 / \rho) U^{0}$ for some $\rho>0$. This means $1 / \rho \leqq \alpha(V, U, M)=\alpha$. This being true for every $\rho>0$ with the property $\bar{M} \subset \rho U$, it is also true with $p(U, \bar{M})$ in place of $\rho$. Note that $p(U, \bar{M})$ is nonzero by hypothesis. Hence $\alpha \geqq 1 / p(U, \bar{M})$.

Again if $\alpha>0$ then from $\mathrm{cl}\left(V \cap M^{0}\right) \supset \alpha U^{0}$ we get $\bar{M} \subset(1 / \alpha) U$. In other words, $p(U, \bar{M}) \leqq 1 / \alpha$ and Lemma 2 follows.

TheOREM 2. Let $\beta(V, U, B)>0$ and $p(U, M) \neq 0$. Then

$$
\begin{aligned}
& \beta(V, U, B) \leqq p(U, M) / p\left(M^{0}, B\right) \\
& \beta(V, U, B) \leqq p(U, M) / p\left(B^{0}, \bar{M}\right) .
\end{aligned}
$$

In particular, $\beta(V, U) \leqq p(U, M) / p(U, \bar{M})$.

Proof. Let $p(U, M)=\lambda$. Write $\beta=\beta(V, U, B)$.

Now $\lambda M^{0} \supset \operatorname{cl}\left(V \cap \lambda M^{0}\right) \supset \mathrm{cl}\left(V \cap U^{0}\right) \supset \beta B$. So $B \subset(\lambda / \beta) M^{0}$ and hence $p\left(M^{0}, B\right) \leqq \lambda / \beta$. 
Again since $\operatorname{cl}\left(V \cap \lambda M^{0}\right) \supset \beta B$ we have $\left(V \cap \lambda M^{0}\right)_{0} \subset(1 / \beta) B_{0}$. This gives $(1 / \lambda) \bar{M} \subset(1 / \beta) B_{0}$ and so $p\left(B_{0}, \bar{M}\right) \leqq \lambda / \beta$.

THEOREM 3. (a) $\beta(V, U) \leqq \alpha(V, U)$.

(b) $\beta(V) \leqq \alpha(V)$.

Proof. It suffices to prove (a). Write $\beta=\beta(V, U)$. Then $\mathrm{cl}\left(V \cap U^{0}\right) \supset \beta U^{0}$. Let $p(U, M)=\lambda \neq 0$. Then $\lambda \operatorname{cl}\left(V \cap M^{0}\right)=\operatorname{cl}\left(V \cap \lambda M^{0}\right) \supset \operatorname{cl}\left(V \cap U^{0}\right) \supset \beta U^{0}$. Therefore $\operatorname{cl}\left(V \cap M^{0}\right) \supset(\beta / \lambda) U^{0}$ and $\alpha(V, U, M) \geqq \beta / \lambda$. This is true for every $M$ for which $p(U, M) \neq 0$. Therefore $\alpha(V, U) \geqq \beta(V, U)$.

THEOREM 4. Let $V$ be a $\tau_{s}(E)$-dense subspace of $E^{\prime}$. Consider the following statements:

(a) $V$ is duxial in $E^{\prime}$.

(b) $\bar{M}$ is bounded in $E[\tau]$ for all $M$.

(c) $p(U, \bar{M})<\infty$ for all $U$ and $M$ for which $p(U, M) \neq 0$.

(d) $\beta(V, U)>0$ for all $U$.

(e) $\alpha(V, U, M)>0$ for all $U$ and $M$ such that $p(U, M) \neq 0$.

(f) $\beta(V)=1$.

(g) $\alpha(V)=1$.

(h) $p(U, M)=p(U, \bar{M})$ for all $U$ and $M$ such that $p(U, M) \neq 0$.

Then we have the following implications:

$$
\begin{array}{ccc}
\text { (a) } \Rightarrow \text { (b) } & \Rightarrow & \text { (c) } \\
& & 1 \\
\text { (d) } & \Rightarrow & (\mathrm{e}) \\
\Uparrow & & \Uparrow \\
\text { (f) } & \Rightarrow & (\mathrm{g}) \Leftrightarrow(\mathrm{h}) .
\end{array}
$$

If $E[\tau]$ is a Mackey space, we have, in addition,

$$
\text { (d) } \Rightarrow \text { (a). }
$$

Proof. In virtue of Theorems 1 and 3 we have to prove only the following implications: (a) $\Rightarrow(\mathrm{b})$ and $(\mathrm{g}) \Leftrightarrow(\mathrm{h})$. The implication (d) $\Rightarrow(\mathrm{a})$ when $\tau$ is the Mackey topology follows from the definition of duxiality.

To prove (a) $\Rightarrow(\mathrm{b})$. Since $U^{0}$ is $\tau_{s}(E)$-compact, we have, by duxiality of $V$, the existence of a $\tau_{b}(E)$-bounded set $F$ such that $U^{0} \subset \operatorname{cl}(V \cap F)$. Now the $\tau_{b}(E)$ boundedness of $F$ gives a $\lambda>0$ such that $F \subset \lambda M^{0}$. Therefore $U \supset(\operatorname{cl}(V \cap F))_{0}$ $=(V \cap F)_{0} \supset\left(V \cap \lambda M^{0}\right)_{0}=(1 / \lambda) \bar{M}$. In other words, $\bar{M} \subset \lambda U$. Since $U$ is arbitrary in $\mathscr{U}$ it follows that $\bar{M}$ is bounded in $E[\tau]$.

To prove (g) $\Rightarrow(\mathrm{h})$. Let $\alpha(V)=1$. This means $\alpha(V, U)=1$ for all $U$. So whenever $U$ and $M$ are such that $p(U, M) \neq 0$, then $\alpha(V, U, M)>0$. Now Theorem 1 shows that for such pairs $U, M, \alpha(V, U, M)=1 / p(U, \bar{M})$. In other words, $1=\alpha(V, U)$ $=\inf \{p(U, M) / p(U, \bar{M}): M \in \mathscr{M}, p(U, M) \neq 0\}$. Therefore $p(U, M)=p(U, \bar{M})$ for all $U$ and $M$ such that $p(U, M) \neq 0$. 
To prove (h) $\Rightarrow(\mathrm{g})$. Let $p(U, M)=p(U, \bar{M})$ for all $U$ and $M$ for which $p(U, M)$ $\neq 0$. Then for such pairs $U, M, p(U, \bar{M})<\infty$ and so $\alpha(V, U, M)=1 / p(U, \bar{M})$. Therefore $\alpha(V, U)=\inf \{p(U, M) / p(U, \bar{M}): M \in \mathscr{M}, p(U, M) \neq 0\}=1$. Hence $\alpha(V)=1$.

THeOREM 5. Let $E$ be canonically embedded in $E^{\prime \prime}$. Then

(a) $\alpha(E)=\beta(E)=1$, and

(b) $E$ is duxial in $E^{\prime \prime}$ if and only if $E^{\prime}\left[\tau_{b}(E)\right]$ is a Mackey space.

REMARK. L.c. spaces $E$ for which $E^{\prime}\left[\tau_{b}(E)\right]$ is a Mackey space are said to be semidistinguished (cf. (I) p. 339 and also Table 2).

Proof. Since the $\tau_{s}\left(E^{\prime}\right)$-closure in $E^{\prime \prime}$ of every $M \in \mathscr{M}$ is $M^{00}$, it follows that $E \cap M^{00}$ is $\tau_{s}\left(E^{\prime}\right)$-dense in $M^{00}$. Now the sets $\left\{M^{0}, M \in \mathscr{M}\right\}$ form a fundamental system of neighbourhoods of zero for $E^{\prime}\left[\tau_{b}(E)\right]$. So $\beta\left(E, M^{0}\right)=1$ for every $M \in \mathscr{M}$ and this means $\beta(E)=1$. It then follows from Theorem 3 that $\alpha(E)$ is also equal to 1 . This proves (a).

To prove (b) we note that (cf. Introduction) duxiality of $E$ in $E^{\prime \prime}$ is equivalent to saying that every convex $\tau_{s}\left(E^{\prime}\right)$-compact subset $K$ of $E^{\prime \prime}$ is contained in the $\tau_{s}\left(E^{\prime}\right)$ closure in $E^{\prime \prime}$ of some bounded subset of $E\left[\tau_{b i}\left(E^{\prime}, E^{\prime \prime}\right)\right]$ that is, of $E\left[\tau_{b^{*}}\left(E^{\prime}, E\right)\right]$. But the bounded subsets of $E\left[\tau_{b^{*}}\left(E^{\prime}\right)\right]$ are the same as the bounded sets of $E\left[\tau_{s}\left(E^{\prime}\right)\right]$ and of $E[\tau]$, since the bornological topology associated with these is bigger than $\tau_{b^{*}}\left(E^{\prime}\right)$. Thus every $K$ is contained in the $\tau_{s}\left(E^{\prime}\right)$-closure in $E^{\prime \prime}$ of some $\tau$-bounded subset of $E$. This, by definition, is equivalent to saying that $E$ is semidistinguished.

REMARK. The example of Komura [2] of a space for which $E^{\prime}\left[\tau_{b}(E)\right]$ is not a Mackey space serves as an example for the situation where $E$ is of characteristic one in $E^{\prime \prime}$ but not duxial in $E^{\prime \prime}$. This therefore shows that for the subspace $E$ of $E^{\prime \prime}$, among the properties mentioned in Theorem 4, (a) is not satisfied even when all the others are.

5. Generalizations of (1.2) and (1.4). For this section we need the following lemma which is perhaps well known, but for which the author has not been able to find any reference.

Lemma 3. (a) For every $U \in \mathscr{U}$, and for all $x \in E, p(U, x)=\sup \left\{|f(x)|: f \in U^{0}\right\}$.

(b) For every $M \in \mathscr{M}$ and for all $f \in E^{\prime}, p\left(M^{0}, f\right)=\sup \{|f(x)|: x \in M\}$.

Proof. Consider the seminorm defined by $x \rightarrow \sup \left\{|f(x)|: f \in U^{0}\right\}$. The unit ball of this seminorm is $\left\{x \in E:\left\{\sup |f(x)|: f \in U^{0}\right\} \leqq 1\right\}$. But this latter is just $U$. Now we know there is a one-to-one correspondence between the seminorms on $E$ on the one hand and on the other hand the absolutely convex absorbing sets whose intersection with every real homogeneous straight line is closed. Hence the seminorm coincides with $p(U, x)$. This proves (a). The proof of $(b)$ is similar.

THEOREM 6. For all $U$ and $M$ such that $p(U, M) \neq 0$,

(a) $\alpha(V, U, M)=\inf \left\{\sup _{f \in V \cap M^{0}}|f(x)| \mid p(U, x): x \in E, p(U, x) \neq 0\right\}$ and

(b) $\beta(V, U)=\inf \left\{\sup _{f \in V \cap U^{0}}|f(x)| / p(U, x): x \in E, p(U, x) \neq 0\right\}$. 
Proof. Write $\alpha=\alpha(V, U, M)$ and $\beta=\beta(V, U)$. We shall prove (a) below. To prove (b) we have only to replace $\alpha$ by $\beta$ and $V \cap M^{0}$ by $V \cap U^{0}$ in the following proof.

Denote by $s$ the infimum on the right side of (a). Let $x_{0} \in E$ with $p\left(U, x_{0}\right) \neq 0$. Let $\varepsilon>0$.

Let $g \in(s-\varepsilon) U^{0}$. Then $\left|g\left(x_{0}\right)\right| \leqq(s-\varepsilon) p\left(U, x_{0}\right)$. Now there exists $f_{0} \in V \cap M^{0}$ such that $\left|f_{0}\left(x_{0}\right)\right| \geqq(s-\varepsilon) p\left(U, x_{0}\right) \geqq\left|g\left(x_{0}\right)\right|$. Write $f_{1}=f_{0} g\left(x_{0}\right) / f_{0}\left(x_{0}\right)$. This $f_{1} \in V$ $\cap M^{0}$ and $f_{1}\left(x_{0}\right)=g\left(x_{0}\right)$. Thus for every $x_{0} \in E$ with $p\left(U, x_{0}\right) \neq 0$ there exists $f_{1} \in \operatorname{cl}\left(V \cap M^{0}\right)$ such that $f_{1}\left(x_{0}\right)=g\left(x_{0}\right)$. In other words $g$ and $\operatorname{cl}\left(V \cap M^{0}\right)$ cannot be separated by such elements $x_{0}$. Clearly $g$ cannot be separated from $\mathrm{cl}\left(V \cap M^{0}\right)$ by elements $x_{0}$ with $p\left(U, x_{0}\right)=0$; for in such a case $g\left(x_{0}\right)=0$. Thus $g \in \operatorname{cl}\left(V \cap M^{0}\right)$. $g$ being arbitrary in $(s-\varepsilon) U^{0}$ we get $(s-\varepsilon) U^{0} \subset \operatorname{cl}\left(V \cap M^{0}\right)$. Hence $\alpha \geqq(s-\varepsilon)$. Since $\varepsilon$ is arbitrary it follows that $\alpha \geqq s$.

On the other hand, by Lemma 3 above, $\alpha p\left(U, x_{0}\right)=\sup \left\{\left|f\left(x_{0}\right)\right|: f \in \alpha U^{0}\right\}$. $\alpha U^{0}$ being $\tau_{s}(E)$-compact there exists $g \in \alpha U^{0}$ such that $\left|g\left(x_{0}\right)\right|=\alpha p\left(U, x_{0}\right)$. Replacing $g$ by $g_{0}=g \exp \left(-i \arg \left(g\left(x_{0}\right)\right)\right)$ we see that there exists $g_{0} \in \alpha U^{0}$ such that $g_{0}\left(x_{0}\right)=\alpha p\left(U, x_{0}\right)$. But we know $\operatorname{cl}\left(V \cap M^{0}\right) \supset \alpha U^{0}$. Therefore $g_{0} \in \operatorname{cl}\left(V \cap M^{0}\right)$. Consider the $\tau_{s}(E)$-neighbourhood of $g_{0}$ defined by

$$
\left\{f:\left|f\left(x_{0}\right)-g\left(x_{0}\right)\right| \leqq \varepsilon p\left(U, x_{0}\right)\right\} .
$$

This contains $f_{0} \in V \cap M^{0}$. Hence $\left|f_{0}\left(x_{0}\right)-g_{0}\left(x_{0}\right)\right| \leqq \varepsilon p\left(U, x_{0}\right)$ and so $\left|f_{0}\left(x_{0}\right)\right|$ $\geqq \alpha p\left(U, x_{0}\right)-\varepsilon p\left(U, x_{0}\right)$. Hence

$$
\sup _{f \in V \cap M^{0}}\left\{\left|f\left(x_{0}\right)\right| / p\left(U, x_{0}\right)\right\} \geqq \alpha-\varepsilon .
$$

$\varepsilon$ being arbitrary and $x_{0}$ being any element of $E$ with $p\left(U, x_{0}\right) \neq 0$, we get $\alpha \leqq s$. This completes the proof of the theorem.

THEOREM 7. Let $U \in \mathscr{U}$ and $V$ be a $\tau_{s}(E)$-dense subspace of $E^{\prime}$. Let $V^{\perp}$ be the orthogonal complement of $V$ in $E^{\prime \prime}$. Then

$$
\beta(V, U)=\inf \left\{p\left(U^{00}, x+z\right) / p(U, x): x \in E, p(U, x) \neq 0, z \in V^{\perp}\right\} .
$$

Proof. Write $\beta$ for $\beta(V, U)$ and $s$ for the infimum on the right hand side. Let $x \in E$ with $p(U, x) \neq 0$ and let $z \in V^{\perp}$. Then

$$
\begin{aligned}
p\left(U^{00}, x+z\right) & =\sup \left\{|(x+z) f|: f \in U^{0}\right\} \\
& \geqq \sup \left\{|(x+z) f|: f \in V \cap U^{0}\right\} \\
& =\sup \left\{|f(x)|: f \in V \cap U^{0}\right\} \\
& =\sup \left\{|f(x)|: f \in \operatorname{cl}\left(V \cap U^{0}\right)\right\} \\
& \geqq \sup \left\{|f(x)|: f \in \beta U^{0}\right\} \\
& =\beta p(U, x) .
\end{aligned}
$$

This gives $s \geqq \beta$. 
On the other hand, given $\varepsilon>0$, there exists $x_{0} \in E$ with $p\left(U, x_{0}\right) \neq 0$ such that

$$
\sup \left\{\left|f\left(x_{0}\right)\right| \mid p\left(U, x_{0}\right): f \in V \cap U^{0}\right\} \leqq \beta+\varepsilon .
$$

Since $E^{\prime \prime} / V^{\perp}$ is the dual of $V\left[\tau_{b i}\left(E, E^{\prime}\right)\right]$ and the natural topology $\tau_{n}\left(E^{\prime}, E^{\prime \prime}\right)$ canonically maps into the topology on $E^{\prime \prime} / V^{\perp}$ of uniform convergence on sets of the form $V \cap U^{0}$ we get

$$
\begin{aligned}
\inf \left\{p\left(U^{00}, x_{0}+z\right): z \in V^{\perp}\right\} & =p\left(U^{00},\left[x_{0}+V^{\perp}\right]\right) \\
& =\sup \left\{\left|\left\langle\left[x_{0}+V^{\perp}\right], f\right\rangle\right|: f \in V \cap U^{0}\right\} \\
& =\sup \left\{\left|\left\langle x_{0}, f\right\rangle\right|: f \in V \cap U^{0}\right\} \\
& \leqq(\beta+\varepsilon) p\left(U, x_{0}\right) .
\end{aligned}
$$

Since $\varepsilon$ is arbitrary, we get $s \leqq \beta$ and this completes the proof.

\section{REFERENCES}

1. J. Dixmier, Sur un théorème de Banach, Duke Math. J. 15 (1948), 1057-1071.

2. K. Komura, Some examples in linear topological spaces, Math. Ann. 153 (1964), 150-162.

3. G. Köthe, Topologische lineare Räume. I, Springer-Verlag, Berlin, 1960.

4. V. Krishnamurthy, Conjugate locally convex spaces, Math. Z. 87 (1965), 334-344.

Birla Institute of Technology and Science, Pilani, Rajasthan, India 